\title{
First-principles momentum distributions and vibrationally corrected permittivities of hexagonal and cubic ice
}

\author{
Edgar A. Engel, Yuting Li, and Richard J. Needs \\ TCM Group, Cavendish Laboratory, J. J. Thomson Avenue, Cambridge CB3 OHE, England, United Kingdom
}

(Received 28 July 2017; published 23 February 2018)

\begin{abstract}
Three-dimensionally resolved proton momentum distributions and end-to-end distributions have been calculated for hexagonal and cubic water ice. First-principles quantum nuclear wave functions have been used to investigate the impact of vibrational anisotropy, anharmonicity, proton and stacking disorder, temperature, and pressure on these distributions. Moreover, the effects of vibrations on the electronic density in hexagonal ice are shown to lead to a $5 \%$ vibrational correction with respect to the static-lattice optical permittivity, and proton disorder is found to be crucial in explaining its experimentally observed temperature dependence.
\end{abstract}

DOI: 10.1103/PhysRevB.97.054312

\section{INTRODUCTION}

Hydrogen-bonded materials play an important role across many fields of science. The importance of water ice, in particular, spans disciplines ranging from astrophysics to biology. Properties of hydrogen-bonded materials are linked to those of the hydrogen bond [1,2], and are strongly affected by the quantum nuclear motion of the light protons [3-7]. Anharmonic quantum nuclear effects play a key role in ice. For example, they stabilize hexagonal ice (Ih) with respect to cubic ice (Ic), which ultimately leads to the hexagonal shape of ice crystals [7]. Anharmonicity also plays an important role in the anomalous thermal expansion of ice Ih [8], in proton and deuteron isotopic effects [9-11], and in shifts in infrared and other vibrational spectra [7]. Quantum zero-point (ZP) and thermal motion of the protons have a large impact on the electronic properties of ice $[12,13]$. Here we study the effects of vibrational motion on the electronic density and permittivity of ice using first-principles density-functional-theory (DFT) methods. Quantum ZP motion dominates the equilibrium proton dynamics of ice up to the melting temperature [12]. Proton dynamics therefore provide a rare direct probe of their quantum nature. We have compared the results of our firstprinciples calculations of position and momentum distributions with experimental data, which provides a stringent test of computational descriptions of ice and the hydrogen bond.

\section{PROTON REAL-SPACE AND MOMENTUM DISTRIBUTIONS}

The proton radial distribution function (RDF) provides a real-space measure of proton dynamics, whereas the momentum distribution function (MDF) gives complementary

Published by the American Physical Society under the terms of the Creative Commons Attribution 4.0 International license. Further distribution of this work must maintain attribution to the author(s) and the published article's title, journal citation, and DOI. reciprocal-space information [14]. Experimentally, the RDF can be accessed by inversion of the neutron [15] and x-ray structure factors [16], while neutron Compton scattering and deep inelastic neutron scattering $[17,18]$ can probe the MDF. Path-integral molecular dynamics (PIMD) simulations have been the method of choice for calculating both the RDF [19-21] and MDF [22-24] and have provided important insights into the RDF and MDF signatures of the interactions of the protons with their environment. However, they have also demonstrated that some of the most commonly used empirical models of water do not accurately reproduce the experimental MDF. Experimental data are currently limited to the spherically averaged MDF and are insufficient to disentangle the effects of environmental anisotropy and vibrational anharmonicity [25]. This gives rise to severe difficulties in interpreting experiments on nanoconfined and supercooled water [26,27]. Such experiments highlight the need for accurate $a b$ initio simulations of proton distribution functions, such as the pathintegral Car-Parinello molecular dynamics (PICPMD) studies of Lin et al. [25] and Flammini et al. [18].

PIMD studies, including those of the PICPMD flavor, have their limitations.

(i) They are expensive, which limits the number (and sizes) of systems, temperatures, and pressures that can be studied.

(ii) The effects of anharmonicity cannot be extracted directly, as PIMD inherently simulates the behavior of the anharmonic system.

(iii) At its core PIMD is a sophisticated phase-space sampling method in which statistical uncertainty limits the accuracy with which (complicated) quantities, such as the spatially resolved MDF, can be determined.

To overcome these limitations, we calculate the MDF directly from the fully anharmonic nuclear wave function that is obtained using the vibrational self-consistent field (VSCF) approach of Monserrat et al. [28]. This provides the unprecedented level of microscopic insight required to disentangle the effects of anisotropy, vibrational anharmonicity, pressure, temperature, and stacking and proton disorder. Our analytic description of the vibrational wave function also greatly facilitates calculations of accurate three-dimensionally 
resolved MDFs. Moreover, the VSCF approach naturally lends itself to the use of nondiagonal supercells [29], which enable extensive sampling of the vibrational Brillouin zone (BZ) without the need for large supercell simulations. This renders the VSCF approach far less expensive than PIMD methods when points in the vibrational $\mathrm{BZ}$ other than the $\Gamma$ point need to be sampled for converged results. Notably, calculations using 64-molecule simulation cells show that $\Gamma$-point calculations using eight-molecule simulation cells suffice in the context of proton RDFs and MDFs in ice Ih and Ic.

\section{A. Computational methods}

We use DFT as implemented in the CASTEP plane-wave pseudopotential package [30] (version 7.03) with the PBE exchange-correlation (xc) functional [31], which reproduces the experimental lattice parameters of ice Ih and Ic rather well [7]. Geometry optimizations and the mapping of the BornOppenheimer potential-energy surface (PES) were performed with a plane-wave energy cutoff of $1600 \mathrm{eV}$, a Monkhorst-Pack k-point grid [32] spacing of less than $2 \pi \times 0.04 \AA^{-1}$, and onthe-fly generated ultrasoft pseudopotentials [33]. The resulting energy differences between frozen-phonon configurations are converged to below $10^{-4} \mathrm{eV} / \mathrm{H}_{2} \mathrm{O}$, the atomic positions are converged to within $10^{-5} \AA$, and the residual forces are converged to within $10^{-4} \mathrm{eV} / \AA$.

For an $N$-atom system, the $3 N$ harmonic vibrational normal modes $(n, \mathbf{K})$ with branch index $n$, first vibrational BZ momentum $\mathbf{K}$, and frequencies $\omega_{n \mathbf{K}}$ are calculated using a finite displacement approach [34]. The harmonic approximation is used to define normal-mode coordinates $q_{n \mathbf{K}}$, which measure the collective atomic displacements along the normal modes $(n, \mathbf{K})$. They are related to the Cartesian displacement of the nucleus $i$ with mass $M_{i}$ from its equilibrium position, $\mathbf{u}_{i}$, by

$$
q_{n \mathbf{K}}=\sum_{i} \sqrt{M_{i}} \exp \left(-i \mathbf{K} \cdot \mathbf{R}_{i}\right) \mathbf{u}_{i} \cdot \mathbf{w}_{-\mathbf{K} n i}
$$

where $\mathbf{w}_{-\mathbf{K} n I \mu}$ is the displacement pattern of mode $(n, \mathbf{K})$. The momentum $\mathbf{K}$ is suppressed in the following as we restrict ourselves to $\Gamma$-point calculations.

The light mass of the protons, $M_{p}=1837.3621238166$ a.u., leads to large displacements due to quantum zero-point motion, implying that anharmonicity in the PES is important. In ice Ih and Ic anharmonic vibrations can be calculated within the independent-mode approximation due to the small coupling between normal modes [7]. Accordingly, the PES is expressed as a function of $\mathbf{q}=\left(q_{i}, \ldots, q_{3 N}\right)$, expanded in independentmode terms $V^{(1)}\left(q_{n}\right)$, pairwise couplings $V^{(2)}\left(q_{n}, q_{n^{\prime}}\right)$, etc., and then truncated after the $V^{(1)}\left(q_{n}\right) \equiv V\left(0, \ldots, q_{n}, \ldots, 0\right)-V(\mathbf{0})$ term:

$$
V(\mathbf{q})=V(\mathbf{0})+\sum_{n} V^{(1)}\left(q_{n}\right)+\frac{1}{2} \sum_{n} \sum_{n^{\prime} \neq n} V^{(2)}\left(q_{n}, \widehat{q_{n^{\prime}}}\right)+\cdots .
$$

The $3 N$ anharmonic independent-mode potentials $V^{(1)}\left(q_{n}\right)$ are mapped up to large amplitudes of four times the harmonic rms displacements and fitted using cubic splines. Mapping each $V^{(1)}\left(q_{n}\right)$ using 11 equally spaced points was found to lead to converged results. The vibronic Schrödinger equation

$$
\left(-\sum_{n} \frac{1}{2} \nabla_{q_{n}}^{2}+V(\mathbf{q})\right)\left|\Psi^{\mathbf{S}}\right\rangle=E^{\mathbf{S}}\left|\Psi^{\mathbf{S}}\right\rangle
$$

is solved following the vibrational self-consistent field method described in Ref. [28]. The anharmonic vibrational wave function is expanded as a product,

$$
\left|\Psi_{\mathbf{S}}(\mathbf{q})\right\rangle=\prod_{n}\left|\psi_{S_{n}}\left(q_{n}\right)\right\rangle,
$$

of single-particle anharmonic eigenstates, $\left|\psi_{S_{n}}\left(q_{n}\right)\right\rangle$, each using a basis of 25 simple harmonic oscillator eigenstates. $\mathbf{S}$ denotes the vibrational eigenstate the elements $S_{n}$ of which label the states of the independent modes.

Notably, the choice of xc functional affects nuclear vibrations in ice Ih and Ic very little for fixed unit-cell parameters [7].

\section{B. Comparison with experiment and PICPMD results}

Using the anharmonic (unless explicitly stated) vibrational wave function, the distribution $n_{i}(\mathbf{p})$ of the momentum $\mathbf{p}$ of proton $i$ can be calculated as the Fourier transform of the onebody reduced density matrix $\rho_{i}\left(\mathbf{r}_{i}, \mathbf{r}_{i}^{\prime}\right)$ :

$$
\begin{aligned}
\rho_{i}\left(\mathbf{r}_{i}, \mathbf{r}_{i}^{\prime}\right) & =\int\left(d^{3} \mathbf{r}_{j \neq i}\right)\left\langle\mathbf{r}_{i}^{\prime} \mid \Psi\right\rangle\left\langle\Psi \mid \mathbf{r}_{i}\right\rangle, \\
n_{i}(\mathbf{p}) & =\int d^{3} \mathbf{r}_{i} d^{3} \mathbf{r}_{i}^{\prime} \exp \left[-\frac{i}{\hbar} \mathbf{p} \cdot\left(\mathbf{r}_{i}-\mathbf{r}_{i}^{\prime}\right)\right] \rho_{i}\left(\mathbf{r}_{i}, \mathbf{r}_{i}^{\prime}\right),
\end{aligned}
$$

where $\int\left(d^{3} \mathbf{r}_{j \neq i}\right)$ denotes integration over the atomic positions $\mathbf{r}_{j}$ of all $3 N$ atoms in the simulation cell except atom $i$. In the following we refer to

$$
n_{i}(\mathbf{r})=\int d^{3} \mathbf{r}_{i} \rho_{i}\left(\mathbf{r}_{i}, \mathbf{r}_{i}+\mathbf{r}\right)
$$

as the "end-to-end distribution" in analogy with open-ended path-integral simulations [35], and the absence of the index $i$ denotes quantities that have been averaged over all protons. In practice $n_{i}(\mathbf{r})$ and $n_{i}(\mathbf{p})$ are both discretized in real and reciprocal space, respectively. Measurements of the spatially resolved MDF, $n(\mathbf{p})$, are not yet available for ice, but the spherically averaged distribution $n_{p}(p) \equiv \int d \theta d \phi p^{2} n(\mathbf{p})$ has been measured, for example, by Reiter et al. [17] and Senesi et al. [36]. Their data for $n_{p}(p)$, the anisotropy of $n(\mathbf{p})$, and the kinetic energy of the protons provide a measure of the accuracy of computational approaches.

The spherically averaged MDF, $n_{p}(p)$, is conventionally described by

$$
n_{p}(p)=\frac{-1}{2 \pi p} \frac{d}{d p}\left[\frac{\exp \left(-\frac{y^{2}}{2 \sigma^{2}}\right)}{\sqrt{2 \pi} \sigma} \sum_{n} \frac{a_{n}}{2^{2 n} n !} H_{2 n}\left(\frac{y}{\sqrt{2} \sigma}\right)\right],
$$

where $\sigma$ and the dimensionless $a_{n}$ are fitting parameters and the $H_{n}$ are the usual Hermite polynomials. The widths $\sigma$ obtained in this paper and listed in Table I agree well with recent experimental [18] and PICPMD [25] data. We find that the kinetic energy of the protons $T_{\mathrm{p}}$, which can be calculated as $T_{\mathrm{p}}=\int_{0}^{\infty} p^{2} /(2 M) n_{p}(p) d p$ or (within the 
TABLE I. The kinetic energies of the protons $T_{\mathrm{p}}^{\sigma}=3 \hbar^{2} /(2 M) \sigma^{2}$ are calculated from the estimated width $\sigma$ of the MDF obtained by fitting Eq. (7) to $n_{p}(p)$ at $268 \mathrm{~K}$, while $T_{\mathrm{p}}$ denotes direct, nonparametric estimates of the kinetic energy of the protons. $\sigma_{\|}$and $\sigma_{\perp}$ measure the widths of $n$ (p) parallel and perpendicular to the $\mathrm{O}-\mathrm{H}$ direction, averaged over proton orderings. Errors arise from fitting Eq. (8) to noisy data due to the experimental resolution (Exp), statistical uncertainty from finite sampling (PICPMD), and discretization of $n(\mathbf{r})$ and $n(\mathbf{p})$ (VSCF), respectively.

\begin{tabular}{|c|c|c|c|c|c|c|c|c|}
\hline & $\sigma\left(\AA^{-1}\right)$ & $a_{2}$ & $a_{3}$ & $T_{\mathrm{p}}^{\sigma}(\mathrm{meV})$ & $T_{\mathrm{p}}(\mathrm{meV})$ & $\sigma_{\|}\left(\AA^{-1}\right)$ & $\sigma_{\perp}\left(\AA^{-1}\right)$ & $\sigma_{\|} / \sigma_{\perp}$ \\
\hline Exp [17] & 4.58 & $0.060(1)$ & $-0.07(2)$ & & & $6.29 \pm 0.51$ & $3.53 \pm 0.31$ & $1.8 \pm 0.3$ \\
\hline Exp [18] & 4.99 & $0.064(2)$ & $0.00(1)$ & $156(2)$ & $156(9)$ & $6.47 \pm 0.11$ & $4.30 \pm 0.19$ & $1.5 \pm 0.1$ \\
\hline Harmonic VSCF & 4.85 & $0.057(1)$ & $0.00(1)$ & $147(1)$ & & $6.58 \pm 0.02$ & $3.89 \pm 0.02$ & $1.7 \pm 0.1$ \\
\hline Anharmonic VSCF & 4.96 & $0.063(1)$ & $0.00(1)$ & $154(1)$ & & $6.79 \pm 0.02$ & $4.03 \pm 0.15$ & $1.7 \pm 0.1$ \\
\hline PICPMD [18,25] & 4.98 & & & $155(2)$ & $143(2)$ & $6.28 \pm 0.12$ & $4.18 \pm 0.14$ & $1.5 \pm 0.1$ \\
\hline
\end{tabular}

harmonic approximation) via $T_{\mathrm{p}}=3 \hbar^{2} /(2 M) \sigma^{2}$, matches the experimental and PICPMD data $[18,25]$.

The insensitivity of $n_{p}(p)$ to anisotropy in the momentum distribution popularized the mean force $f(x)$ as a more sensitive probe of the local environment of a proton [35]. Experimentally, $f(x)$ can be extracted via its relationship with the Fourier transform of the neutron Compton profile. Its slope at $x=0$ determines the curvature of the proton potential around the equilibrium position and thereby provides a second measure of the kinetic energy of the protons. A comparison of the mean force obtained using the VSCF vibrational method to that from PICPMD simulations [18] is shown in the inset of Fig. 1.

The anisotropy of $n(\mathbf{p})$ is conventionally described in terms of the widths of the three-dimensional anisotropic Gaussian fit to $n_{i}(\mathbf{p})$ :

$$
n_{i}(\mathbf{p})=\frac{\cos ^{2}\left(\frac{p_{z} d}{2 \hbar}\right)}{1+\exp \left(\frac{-d^{2} \sigma_{z}{ }^{2}}{2 \hbar^{2}}\right)} \prod_{\alpha=x, y, z} \frac{\exp \left(\frac{-p_{\alpha}{ }^{2}}{2 \sigma_{\alpha}{ }^{2}}\right)}{\left(2 \pi \sigma_{\alpha}\right)^{2}},
$$

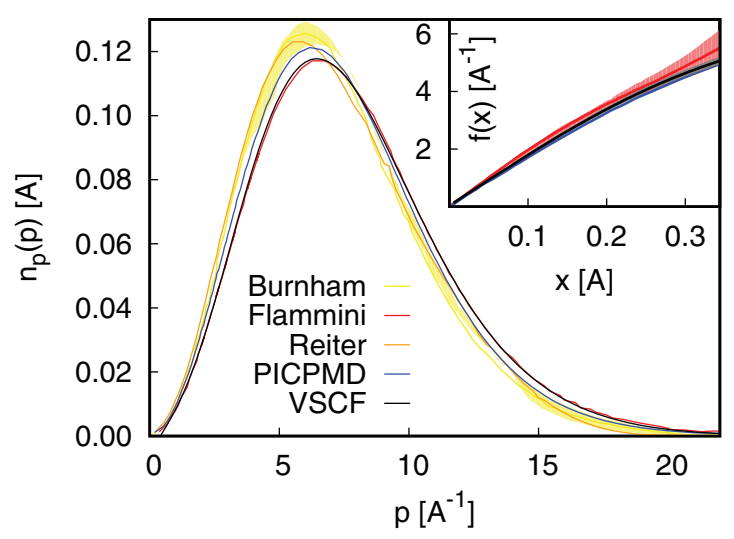

FIG. 1. Comparison of the spherically averaged momentum distribution $n_{p}(p)$ from experiment (yellow [23], orange [17], and red [18]) and path-integral Car-Parinello molecular dynamics (PICPMD) simulations (blue) [18] to VSCF vibrational calculations (black). The inset compares the mean force $f(x)$ with error bars inferred from neutron Compton scattering (red) [18] to PICPMD data (blue) [18] and VSCF calculations. The deviation of $f(x)$ from linearity indicates that the proton potential exhibits substantial anharmonicity. The uncertainties in the experimental distributions arise from fitting models to data with finite experimental resolution. The PICPMD data exhibit small statistical errors, while the VSCF results exhibit negligibly small errors due to the discretization of $n(\mathbf{r})$ and $n(\mathbf{p})$. where $d$ and the widths $\sigma_{\alpha}$ are fitting parameters. The $\mathrm{O}-\mathrm{H} \cdots \mathrm{O}$ axis is characterized by $\sigma_{\|}$, while the two normal axes are typically characterized by a single parameter $\sigma_{\perp}$. The differences between the in- and out-of-molecular plane environments of each proton imply that the two normal axes are inequivalent, and the widths of $n_{i}(\mathbf{p})$ along the normal axes indeed differ by about $10 \%$. Disregarding this, Table I demonstrates that the MDF from the VSCF method is about as good as the PICPMD counterparts, provided vibrational anharmonicity is accounted for. It also demonstrates good agreement with the more recent neutron Compton scattering (NCS) data from Ref. [18], with the exception of the anisotropy of $n(\mathbf{p})$. The differences in $\sigma_{\|}$and $\sigma_{\perp}$ are likely due to the finite number of proton orderings considered.

Measurements of the spatially resolved momentum distribution in $\mathrm{KH}_{2} \mathrm{PO}_{4}$ [37], which in its crystalline form is used in optical modulators and for nonlinear optics such as second-harmonic generation, and $\mathrm{Rb}_{3} \mathrm{H}\left(\mathrm{SO}_{4}\right)_{2}$ [38] suggest that $n_{i}(\mathbf{p})$ in Ih should become accessible in the future with improved experimental techniques.

\section{End-to-end distributions}

In line with Eq. (8), the end-to-end distribution, as defined in Eq. (6), of a proton $i$ in proton-disordered ice is well approximated by an anisotropic Gaussian, $n_{i}(\mathbf{r}) \approx \exp \left(-1 / 2 \mathbf{r}^{T} C_{i}^{-1} \mathbf{r}\right)$ [25], where $C_{i, \alpha, \beta}=\left\langle r_{i, \alpha} r_{i, \beta}\right\rangle$ is the correlation matrix of proton $i$. The principal axes of the anisotropic Gaussian form reflect the molecular orientation of the particular proton. However, in proton-ordered ice the local environments of the protons are generally inequivalent, which is reflected in the end-to-end distribution of individual protons. To highlight the anisotropy of $n_{i}(\mathbf{r})$ and the role of the local proton environment, we factorize $n_{i}(\mathbf{r})$ into an isotropic free-particle contribution and an anisotropic environmental component $\tilde{n}_{i}(\mathbf{r}), n_{i}(\mathbf{r}) \equiv \tilde{n}_{i}(\mathbf{r}) \exp \left(M_{p} k_{B} T \mathbf{r}^{2}\right)$, where $M_{p}$ is the proton mass and $T$ is the temperature. Figure 2(a) shows that $\tilde{n}_{i}(\mathbf{r})$ for an individual proton in Ih $C m c 2_{1}$-the lowest energy proton ordering known in Ih-deviates substantially from the purely anisotropic Gaussian form and reflects, in particular, the positions of the next-nearest-neighbor protons. Moreover, in a particular proton-ordered configuration of $\mathrm{Ih}$, such as $C m c 2_{1}$, not all possible environments are realized, so that even the proton-averaged $\tilde{n}(\mathbf{r})$ shown in Fig. 2(b) deviates from the hexagonal symmetry expected from an anisotropic Gaussian form [25]. Averaging over the 16 eight-molecule proton orderings of Ih used by Hirsch and Ojamäe [39] as 
(a)

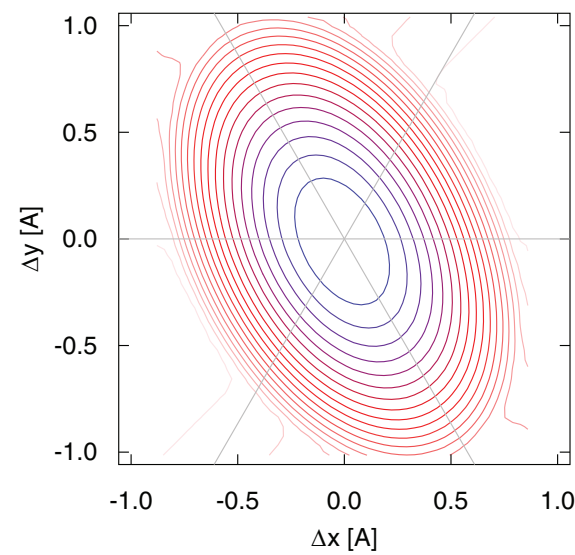

(b)

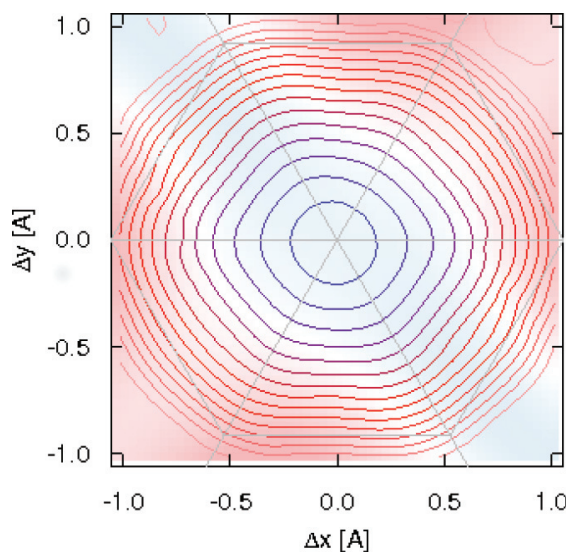

(c)

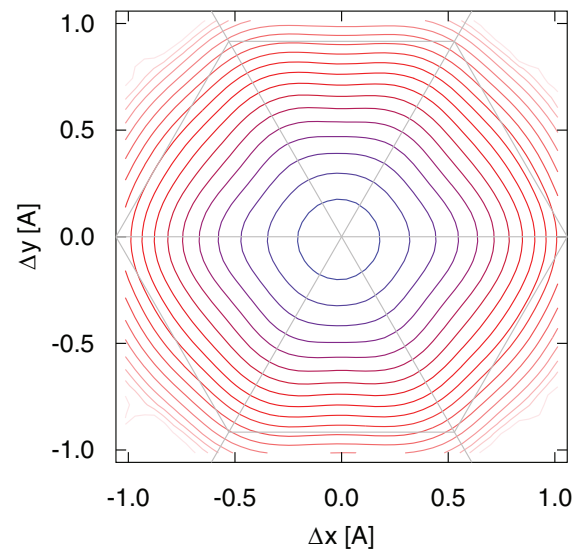

FIG. 2. Projection of the proton environmental end-to-end distributions $\tilde{n}$ (r) onto the basal plane (a) of a single proton, (b) averaged over all protons in Ih $C m c 2_{1}$ (low-temperature, proton-ordered form of Ih), and (c) averaged over all protons in the 16 eight-molecule proton orderings of Hirsch and Ojamäe [39]. In (b) blue and red shading indicate larger and smaller densities with respect to the $\tilde{n}(\mathbf{r})$ of proton-disordered Ih shown in (c), respectively. This highlights deviations from hexagonal symmetry due to the small number of local proton environments realized in Ih $C m c 2_{1}$, irrespective of simulation cell size. Ih $C m c 2_{1}$ simulation cells containing eight and 64 molecules, respectively, exhibit practically identical $\tilde{n}(\mathbf{r})$.

shown in Fig. 2(c) recovers the hexagonal symmetry expected from proton-disordered Ih.

\section{Stacking and proton disorder and vibrational anharmonicity}

As shown in Fig. 3(a) the proton momentum distributions of different proton orderings of Ih are indistinguishable. The differences between the structures of Ih and Ic are very small but are reflected even in the spherically averaged MDFs. The stronger localization of the protons in Ic evidenced by $2 \%$ smaller rms displacements [7] manifests itself in a MDF that is around $2 \%$ wider in Ic than in Ih. Real ice "Ic" samples are typically stacking disordered [40,41]. They contain both Ih and Ic, which differ only in the stacking of molecular layers and which form the end members of the infinite set of stacking-disordered structures generated by introducing stacking faults into the ground-state structure Ih. The MDFs of Ih and Ic thus limit the effects of stacking disorder on the spherically averaged proton MDFs of real ice samples.

The rms proton displacements of Ref. [7], moreover, indicate that vibrational anharmonicity localizes the protons in Ih by around $2 \%$ with respect to the harmonic proton density distribution, suggesting that vibrational anharmonicity should widen the distribution $n(\mathbf{p})$. Figure 3(b) shows that the anharmonic $n_{p}(p)$ is indeed about $4 \%$ wider than its harmonic counterpart. The corresponding harmonic and anharmonic $n(\mathbf{p})$ in the basal and prism planes are shown in Figs. 3(c)-3(d). While Figs. 3(c) and 3(d) highlight the symmetry of $n(\mathbf{p})$, Figs. 3(e) and 3(f) highlight that $n(\mathbf{p})$ widens predominantly in the $\mathrm{O}-\mathrm{H}$ bond direction.

Overall, the differences between the MDFs of different proton orderings of Ih and Ic are too small to distinguish in experiment, but the effects of vibrational anharmonicity, while still small, are already of comparable size to the uncertainty in recent NCS data [18]. This suggests that it should be possible to discern them in future experiments.

\section{E. Temperature and pressure dependence}

The effects of vibrational anharmonicity and stacking disorder on $n_{p}(p)$ are small, begging the question of the roles played by temperature, thermal expansion, and pressure.

In ice only the softest modes, i.e., pseudotranslations, are thermally excited up to melting. Their contribution to proton motion is small, so that any temperature dependence must arise from thermal expansion. The volume expansion of Ih between zero temperature and melting is around $2 \%$. However, neither $n_{p}(p)$ nor $n(\mathbf{p})$ exhibits any discernible temperature dependence across this temperature range.

Conversely, external pressures of up to $\sim 200 \mathrm{MPa}$ lead to volume compressions of up to $1.8 \%$, at which point the Ih-II or Ih-III transition occurs. However, $n_{p}(p)$ and $n(\mathbf{p})$ show no significant pressure dependence, even though the rms proton displacements decrease by up to $0.5 \%$.

The insensitivity of $n_{p}(p)$ to pressure and temperature arises from the same principle: while pseudotranslations and librational modes soften upon expansion, the molecular modes harden, leading to cancellation of their effects on proton motion. Evidence of this is provided in the Supplemental Material (SM) [42].

\section{VIBRATIONAL RENORMALIZATION OF ELECTRONIC PROPERTIES}

Studies of vibrational corrections to band gaps of bulk ice Ih and Ic [12] and their surface band gaps [13] demonstrate that the electronic density is substantially renormalized by quantum nuclear motion. We make use of the explicit form of the anharmonic nuclear wave function provided by the VSCF approach and the adiabatic approximation to sample the electronic density, Born effective charges, and permittivity using Monte Carlo methods.

Within the Born-Oppenheimer approximation, expectation values of vibrationally renormalized properties, $O(T)$, at 


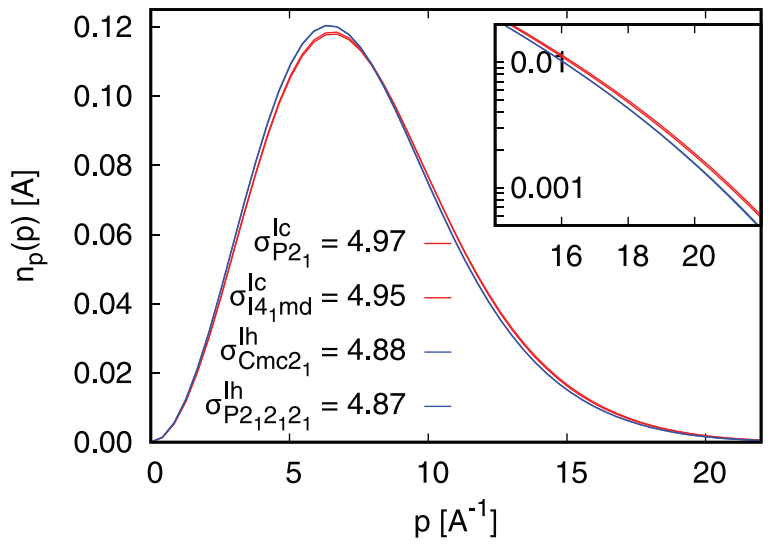

(a)

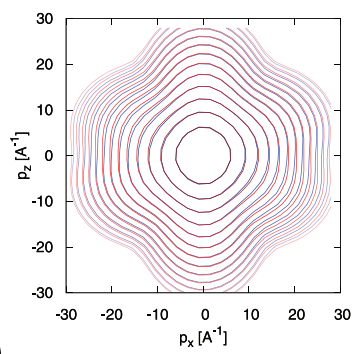

(c)

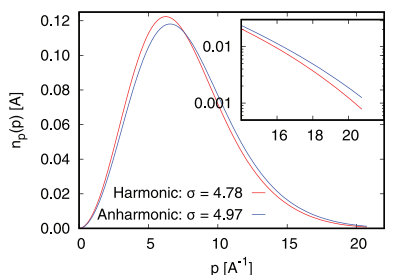

(d)

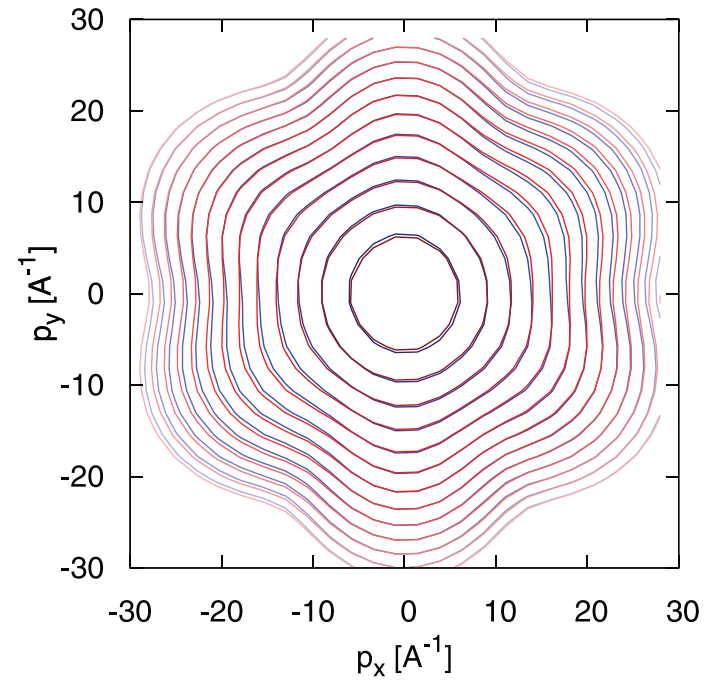

(b)

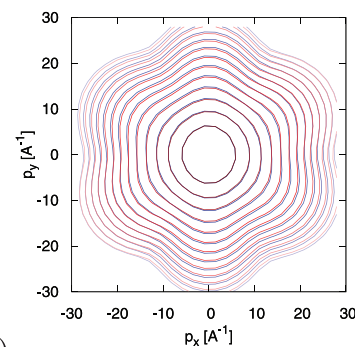

(e)

(f)

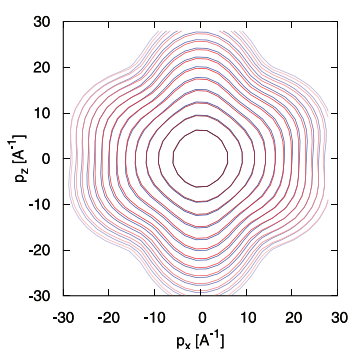

FIG. 3. Panel (a) shows the (anharmonic) $n_{p}(p)$ for different Ih (blue) and Ic (red) proton orderings. Panel (b) shows the harmonic (blue) and anharmonic (red) $n_{p}(p)$ of Ih $C m c 2_{1}$. Panels (c) and (d) show the harmonic (blue) and anharmonic (red) $n$ (p) of Ih $C m c 2_{1}$ projected onto the basal and prism plane, respectively, while panels (e) and (f) show the corresponding anharmonic corrections to $n(\mathbf{p})$.

temperature $T$ may be written as

$$
O(T)=\frac{1}{Z(T)} \sum_{\mathbf{S}}\left\langle\Psi^{\mathbf{S}}(\mathbf{q})|\hat{O}(\mathbf{q})| \Psi^{\mathbf{S}}(\mathbf{q})\right\rangle e^{-\beta E^{\mathbf{S}}}
$$

where

$$
Z(T)=\sum_{\mathbf{S}}\left\langle\Psi^{\mathbf{S}}(\mathbf{q}) \mid \Psi^{\mathbf{S}}(\mathbf{q})\right\rangle e^{-\beta E^{\mathrm{S}}}, \beta=\frac{1}{k_{\mathrm{B}} T}
$$

is the partition function, and $\hat{O}_{g}(\mathbf{q})$ is the value of observable $O$ for a frozen-phonon structure with atomic positions $\mathbf{q}=$ $\left(\ldots, q_{n \mathbf{k}}, \ldots\right)$. The summation over vibrational eigenstates $\mathbf{S}$ includes the ground and excited states.

Here we use Monte Carlo sampling of $O(T)$ with $N_{s}$ frozenphonon structures, $\left\{\mathbf{q}^{i}\right\}$, randomly drawn from the vibrational density, as $1 / Z(T) \sum_{\mathbf{S}}\left|\Psi^{\mathbf{S}}(\mathbf{q})\right|^{2} e^{-\beta \mathrm{E}^{\mathbf{S}}}$, which gives

$$
O(T)=\frac{1}{N_{s}} \sum_{i=1}^{N_{s}} \hat{O}\left(\mathbf{q}^{i}\right)
$$

We initially sample the electronic density, Born effective charges, and permittivity using the harmonic (har) vibrational density at a high temperature $T^{\prime}=260 \mathrm{~K}$, and then obtain the harmonic or (where explicitly stated) anharmonic (anh) expectation values at lower temperatures $T$ using

$$
\begin{aligned}
O(T) & =\frac{1}{N_{s}} \sum_{i=1}^{N_{s}} w_{i}\left(\mathbf{q}^{i}, T\right) \hat{O}\left(\mathbf{q}_{\mathrm{har}}^{i}\right), \\
w_{i}\left(\mathbf{q}^{i}, T\right) & =\frac{Z_{\mathrm{har}}\left(T^{\prime}\right)}{Z(T)} \frac{\sum_{\mathbf{S}}\left|\Psi^{\mathbf{S}}(\mathbf{q})\right|^{2} \mathrm{e}^{-\beta E^{\mathbf{s}}}}{\sum_{\mathbf{S}}\left|\Psi_{\mathrm{har}}^{\mathbf{S}}(\mathbf{q})\right|^{2} \mathrm{e}^{-\beta^{\prime} E_{\mathrm{har}}^{\mathbf{S}}},}
\end{aligned}
$$

where $\beta^{\prime}=1 /\left(k_{\mathrm{B}} T^{\prime}\right)$. This reweighting approach is accurate, because the target vibrational density distributions are narrower than the harmonic distribution at $260 \mathrm{~K}$, from which the frozen-phonon samples are drawn originally.

In practice at least 350 frozen-phonon configurations $\mathbf{q}_{i}$ were sampled to determine various properties for each proton ordering, reducing statistical uncertainties to less than $0.5 \%$. By considering the same frozen-phonon configurations for different functionals, five configurations using the hybrid HSE06 functional $[43,44]$ and about 20 configurations for each additional semilocal functional other than PBE are sufficient to gain insight into the role of the xc functional. More detail regarding the choice of functional can be found in the SM [42].

\section{A. Vibrationally corrected electronic density}

As shown in Fig. 4, nuclear vibrational motion reduces the valence electron density, in particular, around the equilibrium proton positions and the $\mathrm{O}-\mathrm{H}$ covalent bonds. The dominant 


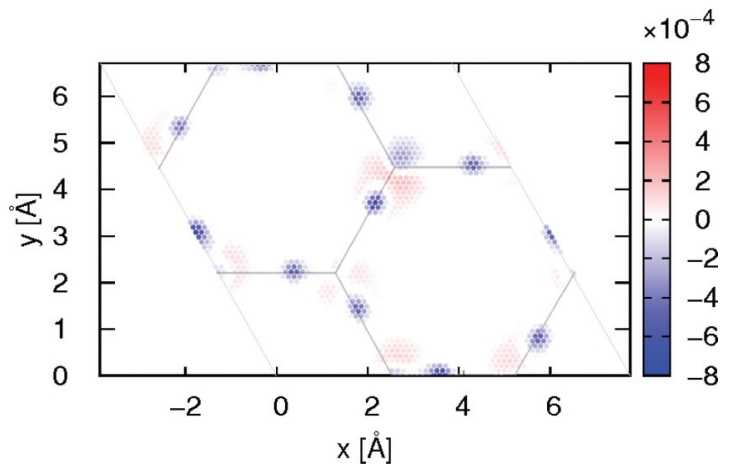

FIG. 4. Vibrational corrections to the static-lattice valence electron density in electrons per unit cell. The oxygen sublattice is indicated by solid gray lines. The large amplitude vibrational motion of the protons, in particular, smears out the electron-density localized on the equilibrium proton positions and on the hydrogen bridge bonds.

(librational/O-H bond bending) motion of the protons is normal to the respective $\mathrm{O}-\mathrm{H} \cdots \mathrm{O}$ axes and delocalises the electronic density around the $\mathrm{O}-\mathrm{H} \cdots \mathrm{O}$ axes.

Delocalization of the valence electron density due to quantum nuclear motion has a striking effect not only on the valenceband maximum in ice and its electronic quasiparticle band gap [12] but also on the electronic and nuclear polarizability of ice. The nuclear polarizability, in particular, is affected by the renormalization of the Born effective charges, $Z^{\star}$. The dependence of $Z^{\star}\left(q_{i}\right)$ on the displacement along a particular vibrational mode, $q_{i}$, is typically dominated by a linear term, which does not contribute to the renormalization of $Z^{\star}$ due to the symmetry of the vibrational wave function. Moreover, calculating $Z^{\star}\left(q_{i}, q_{j}\right)$ for simultaneous displacements $q_{i}, q_{j}$ along pairs of vibrational modes suggests that cross-terms play a negligible role. Instead, the subdominant, higher-order terms in $Z^{\star}\left(q_{i}\right)$ lead to a significant renormalization of $Z^{\star}$, which is reflected in the increase in the nuclear polarizability by around $10 \%$ due to ZP nuclear vibrations. Evidence of this is supplied in the SM [42].

Vibrational corrections to the core electron density around the oxygen nuclei are too small to be resolved in the coarsegrained Fig. 4 due to the small rms displacements of the oxygen nuclei and affect neither the electronic quasiparticle band gap nor the polarizability of ice.

\section{B. Vibrationally corrected permittivity}

The experimentally observed permittivity, $\epsilon$, of ice samples which contain various point and stacking defects exhibits a nontrivial dispersion. The low-frequency behavior around $50 \mathrm{~Hz}$ arises from proton (dis-)order and Bjerrum and/or ionic defects. At intermediate frequencies between around $1 \mathrm{MHz}$ and around $0.3 \mathrm{THz}$ the relative permittivity is roughly constant at a value of around 3.2, and is well described as the sum of the electronic response and the Debye relaxation of the dipoles of the water molecules. Above $0.3 \mathrm{THz}$ crystal vibrational modes (or "pseudotranslations") begin to be excited, although the onset of the nuclear vibrational response occurs with the excitation of librational modes at above $10 \mathrm{THz}$. The nuclear vibrational response determines the detailed permittivity of ice up to around $100 \mathrm{THz}$ before dying off, leaving only the electronic contribution to the permittivity of ice of around 1.7 arising from its electronic polarizability.

Here we focus on the permittivity of ice in the intermediatefrequency regime between $1 \mathrm{MHz}$ and $10 \mathrm{THz}$, which we label simply as $\epsilon$, although the electronic contribution to the permittivity provides insight into the optical permittivity of ice between $400 \mathrm{THz}$ (red light) and $700 \mathrm{THz}$ (blue light). The intermediate-frequency and optical regimes of the permittivity of ice play an important role, for example, in modeling cloud radiative processes and microwave propagation in atmospheric physics/climate science, as well as cloud measurements [45].

Recent work has probed both the permittivity of ice up to the THz regime [46] and its temperature dependence between 190 and $260 \mathrm{~K}$ [47]. These experimental data provide reference points for computation and provide crucial information for understanding the role of proton disorder in determining the permittivity of ice.

Here, the permittivities of the Monte Carlo sampled frozenphonon configurations were calculated within the framework of Ref. [48] and using the CASTEP code to evaluate * and $\epsilon$ in the high-frequency limit, $\epsilon^{\infty}$.

Figure S5 in the Supplemental Material [42] shows $\epsilon$ for the 16 eight-molecule proton orderings of Hirsch and Ojamäe [39] and demonstrates that $\epsilon$ (unlike, for example, the electronic band gap [12]) depends sensitively on the particular proton ordering. In contrast, the increase in $\epsilon$ due to nuclear motion is very similar for all proton orderings and is larger than 5\% of the static-lattice value in all cases considered.

The temperature dependence of $\epsilon$ for a particular proton ordering of Ih is negligible in comparison to the differences between values of $\epsilon$ of up to $18 \%$ among proton orderings. The permittivity of a particular proton ordering generally changes by no more than $0.5 \%$ from zero temperature up to melting. This contrasts with the experimental increase in permittivity of proton-disordered ice of close to $2 \%$ over the same temperature range [46]. Boltzmann averaging over the 16 eight-molecule proton orderings considered results in a temperature-dependent $\epsilon$ which compares reasonably well with experiment (see Fig. 5), although it does not exhibit the clean quadratic increase of the experimental data. This is likely due to (a) the finite set of proton orderings used [49] and (b) uncertainties in proton-ordering energetics. Nevertheless, Fig. 5(a) highlights the importance of proton disorder and thermally induced changes in proton order.

In contrast to the total $\epsilon\left(=\epsilon_{\text {elec }}+\epsilon_{\text {dipole }}\right)$, the electronic contribution to the relative permittivity, $\epsilon_{\text {elec}}$, of 1.87 (exp: $1.72 \pm 0.02$ [50]) is insensitive to the particular proton ordering. $\epsilon_{\text {elec }}$ differs by less than $0.3 \%$ between different proton orderings. The differences in the total $\epsilon$ arise from differences in the dipole contribution, $\epsilon_{\text {dipole }}$, between different proton orderings. Also, $\epsilon_{\text {elec }}$ has a negligibly small intrinsic temperature dependence, irrespective of the particular proton ordering, so that $\epsilon_{\text {elec }}$ exhibits a negligible temperature dependence both for proton-ordered and proton-disordered ice.

Analogously to the mean $\epsilon$, its anisotropy, $\Delta \epsilon \equiv \epsilon^{\|}-\epsilon^{\perp}$, where $\epsilon^{\|}$and $\epsilon^{\perp}$ denote the permittivity along the principal axes parallel and orthogonal to the $c$ axis, varies substantially across proton orderings and exhibits a negligible temperature dependence. Again, the Boltzmann average of the values of $\Delta \epsilon$ 

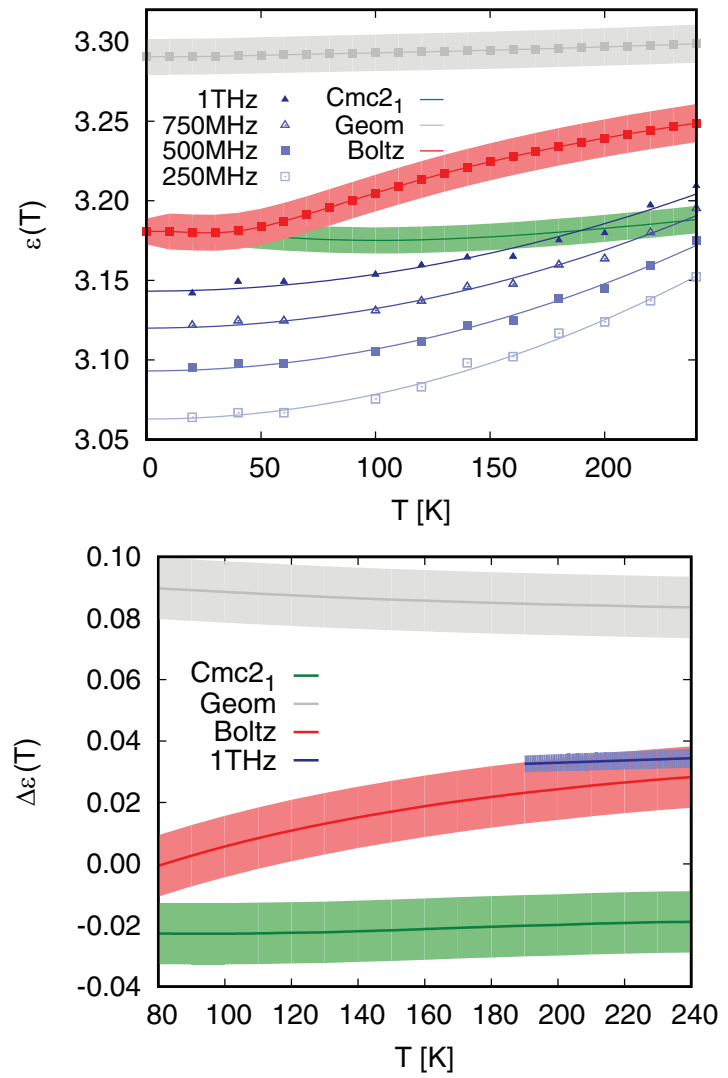

FIG. 5. Comparison of the computed temperature dependence of (a) $\epsilon$ and (b) the anisotropy of $\epsilon, \Delta \epsilon$, for proton-ordered $\left(C m c 2_{1}\right)$ and disordered Ih to experimental $\mathrm{THz}$ data (blue) $[46,47]$. The geometric average (Geom), $\frac{1}{16} \sum_{i=1}^{16} \epsilon^{i}$, and Boltzmann average (Boltzmann), $\frac{1}{Z} \sum_{i=1}^{16} e^{-\beta E^{i}} \epsilon^{i}$, of the $\epsilon^{i}$ of the different proton orderings of Ih are shown in gray and red, respectively. Here, $\beta=1 /\left(k_{B} T\right), Z=$ $\sum_{i=1}^{16} e^{-\beta E^{i}}$, and $E^{i}$ are the configurational energies of the different proton orderings, which differ by up to around $10 \mathrm{meV} / \mathrm{H}_{2} \mathrm{O}$. The shaded regions indicate the statistical uncertainty from Monte Carlo sampling and the experimental uncertainty due to sample preparation, respectively.

compares reasonably well with the data for proton-disordered ice of Fujita et al. [47], as shown in Fig. 5(b).

Both the static-lattice $\epsilon$ and its vibrational correction are sensitive to the choice of $\mathrm{xc}$ functional. However, by constrain- ing the simulation cell volume to the experimental value we largely eliminate the dependence on the xc functional. The role of the xc functional is further investigated in the Supplemental Material [42].

\section{CONCLUSIONS}

Proton disorder, unlike temperature and pressure, substantially affects the proton position and momentum distributions in Ih. It is also crucial for the temperature dependence of the permittivity of Ih. Accurate static-lattice permittivities can be calculated for a particular proton ordering using theoretical methods such as $G W$ many-body perturbation theory [51], or quantum chemical methods, but the 5\% increase in the permittivity of Ih due to nuclear vibrational motion makes it imperative to account for vibrational effects when comparing to experiment.

The VSCF method provides a detailed understanding of the roles of anisotropy and vibrational anharmonicity for the proton momentum distribution, which will facilitate the interpretation of experiments, for example, on nanoconfined and supercooled water. Anharmonic vibrations are crucial in understanding the relative stability of Ih and Ic and many other phenomena. The VSCF vibrational method enables us to directly study their signatures in position and momentum distributions. Finally, the VSCF vibrational method provides the basis for efficient calculations of accurate position and momentum distributions and the means to understand the equilibrium proton dynamics in technologically important materials, such as $\mathrm{KH}_{2} \mathrm{PO}_{4}$ [37] and other hydrogen-bonded materials which are considered candidates for cheap, environmentally friendly, organic electronics [52-54].

\section{ACKNOWLEDGMENTS}

We acknowledge financial support from the Engineering and Physical Sciences Research Council of the United Kingdom (Grant No. EP/P034616/1). The calculations were performed on the Cambridge High Performance Computing Service facility and the Archer facility of the United Kingdom's national high-performance computing service [for which access was obtained via the United Kingdom Car-Parrinello consortium (Grant No. EP/P022596/1)].
[1] X. Li, B. Walker, and A. Michaelides, Quantum nature of the hydrogen bond, Proc. Natl. Acad. Sci. USA 108, 6369 (2011).

[2] R. H. McKenzie, C. Bekker, B. Athokpam, and S. G. Ramesh, Effect of quantum nuclear motion on hydrogen bonding, J. Chem. Phys. 140, 174508 (2014).

[3] D. M. Wilkins, D. E. Manolopoulos, S. Pipolo, D. Laage, and J. T. Hynes, Nuclear quantum effects in water reorientation and hydrogen-bond dynamics, J. Phys. Chem. Lett. 8, 2602 (2017).

[4] B. Monserrat, E. A. Engel, and R. J. Needs, Giant electronphonon interactions in molecular crystals and the importance of nonquadratic coupling, Phys. Rev. B 92, 140302(R) (2015).

[5] V. Srinivasan and D. Sebastiani, The isotope-effect in the phase transition of $\mathrm{KH}_{2} \mathrm{PO}_{4}$ : New insights from ab ini- tio path-integral simulations, J. Phys. Chem. C 115, 12631 (2011).

[6] K. T. Wikfeldt and A. Michaelidis, Communication: $A b$ initio simulations of hydrogen-bonded ferroelectrics: Collective tunneling and the origin of geometrical isotope effects, J. Chem. Phys. 140, 041103 (2014).

[7] E. A. Engel, B. Monserrat, and R. J. Needs, Anharmonic Nuclear Motion and the Relative Stability of Hexagonal and Cubic Ice, Phys. Rev. X 5, 021033 (2015).

[8] B. Pamuk, J. M. Soler, R. Ramírez, C. P. Herrero, P. W. Stephens, P. B. Allen, and M. V. Fernandez-Serra, Anomalous Nuclear Quantum Effects in Ice, Phys. Rev. Lett. 108, 193003 (2012). 
[9] R. Ramírez and C. P. Herrero, Quantum path integral simulation of isotope effects in the melting temperature of ice Ih, J. Chem. Phys. 133, 144511 (2010).

[10] C. P. Herrero and R. Ramírez, Isotope effects in ice Ih: A pathintegral simulation, J. Chem. Phys. 134, 094510 (2011).

[11] L. H. de la Peña, M. S. G. Razul, and P. G. Kusalik, Quantum effects in ice Ih, J. Chem. Phys. 123, 144506 (2005).

[12] E. A. Engel, B. Monserrat, and R. J. Needs, Vibrational renormalisation of the electronic band gap in hexagonal and cubic ice, J. Chem. Phys. 143, 244708 (2016).

[13] E. A. Engel, B. Monserrat, and R. J. Needs, Vibrational effects on surface energies and band gaps in hexagonal and cubic ice, J. Chem. Phys. 145, 044703 (2016).

[14] M. Tuckerman, lecture notes on statistical mechanicslecture 17, New York University, New York City, New York, 1999, http://www.nyu.edu/classes/tuckerman/stat.mech/ lectures/lecture_17/lecture_17.html.

[15] A. Soper, The radial distribution functions of water and ice from 220 to $673 \mathrm{~K}$ and at pressures up to $400 \mathrm{MPa}$, Chem. Phys. 258, 121 (2000).

[16] A. K. Soper, Joint structure refinement of x-ray and neutron diffraction data on disordered materials: Application to liquid water, J. Phys.: Condens. Matter 19, 335206 (2007).

[17] G. F. Reiter, J. C. Li, J. Mayers, T. Abdul-Redah, and P. Platzman, The proton momentum distribution in water and ice, Braz. J. Phys. 34, 142 (2004).

[18] D. Flammini, A. Pietropaolo, R. Senesi, C. Andreani, F. McBride, A. Hodgson, M. A. Adams, L. Lin, and R. Car, Spherical momentum distribution of the protons in hexagonal ice from modeling of inelastic neutron scattering data, J. Chem. Phys. 136, 024504 (2012).

[19] S. Habershon, T. E. Markland, and D. E. Manolopoulos, Competing quantum effects in the dynamics of a flexible water model, J. Chem. Phys. 131, 024501 (2009).

[20] C. P. Herrero and R. Ramírez, High-density amorphous ice: A path-integral simulation, J. Chem. Phys. 137, 104505 (2012).

[21] N. Sato and K. Kinugawa, Path integral molecular dynamics simulation on atomic distribution in amorphized ice ic, Natural Science 8, 460 (2016).

[22] C. J. Burnham, G. F. Reiter, J. Mayers, T. Abdul-Redah, H. Reichert, and H. Dosch, On the origin of the redshift of the $\mathrm{OH}$ stretch in Ice Ih: Evidence from the momentum distribution of the protons and the infrared spectral density, Phys. Chem. Chem. Phys. 8, 3966 (2006).

[23] C. J. Burnham, D. J. Anick, P. K. Mankoo, and G. F. Reiter, The vibrational proton potential in bulk liquid water and ice, J. Chem. Phys. 128, 154519 (2008).

[24] J. A. Morrone, V. Srinivasan, D. Sebastiani, and R. Car, Proton momentum distribution in water: And open path integral molecular dynamics study, J. Chem. Phys. 126, 234504 (2007).

[25] L. Lin, J. A. Morrone, R. Car, and M. Parinello, Momentum distribution, vibrational dynamics, and the potential of mean force in ice, Phys. Rev. B 83, 220302 (2011).

[26] G. Reiter, C. Burnham, D. Homouz, P. M. Platzman, J. Mayers, T. Abdul-Redah, A. P. Moravsky, J. C. Li, C.-K. Loong, and A. I. Kolesnikov, Anomalous Behaviour of Proton Zero Point Motion in Water Confined in Carbon Nanotubes, Phys. Rev. Lett. 97, 247801 (2006).

[27] G. F. Reiter and A. Deb, The quantum mechanics of nanoconfined water: New cooperative effects revealed with neutron and x-ray compton scattering, J. Phys.: Conf. Ser. 571, 012001 (2014).

[28] B. Monserrat, N. D. Drummond, and R. J. Needs, Anharmonic vibrational properties in periodic systems: Energy, electronphonon coupling, and stress, Phys. Rev. B 87, 144302 (2013).

[29] J. H. Lloyd-Williams and B. Monserrat, Lattice dynamics and electron-phonon coupling calculations using nondiagonal supercells, Phys. Rev. B 92, 184301 (2015).

[30] S. J. Clark, M. D. Segall, C. J. Pickard, P. J. Hasnip, M. I. J. Probert, K. Refson, and M. C. Payne, First principles methods using CASTEP, Z. Kristallogr. 220, 567 (2005).

[31] J. P. Perdew, K. Burke, and M. Ernzerhof, Generalized Gradient Approximation Made Simple, Phys. Rev. Lett. 77, 3865 (1996).

[32] H. J. Monkhorst and J. D. Pack, Special points for Brillouin-zone integrations, Phys. Rev. B 13, 5188 (1976).

[33] D. Vanderbilt, Soft self-consistent pseudopotentials in a generalized eigenvalue formalism, Phys. Rev. B 41, 7892(R) (1990).

[34] K. Kunc and R. M. Martin, Ab Initio Force Constants of GaAs: A New Approach to Calculation of Phonons and Dielectric Properties, Phys. Rev. Lett. 48, 406 (1982).

[35] L. Lin, J. A. Morrone, R. Car, and M. Parrinello, Displaced Path Integral Formulation for the Momentum Distribution of Quantum Particles, Phys. Rev. Lett. 105, 110602 (2010).

[36] R. Senesi, A. I. Kolesnikov, and C. Andreani, Measurement of proton momentum distributions using a direct geometry instrument, J. Phys.: Conf. Ser. 571, 012007 (2014).

[37] G. F. Reiter, J. Mayers, and P. Platzman, Direct Observation of Tunneling in KDP using Neutron Compton Scattering, Phys. Rev. Lett. 89, 135505 (2002).

[38] D. Homouz, G. Reiter, J. Eckert, J. Mayers, and R. Blinc, Measurement of the 3D Born-Oppenheimer Potential of a Proton in a Hydrogen-Bonded System using Deep Inelastic Neutron Scattering: The Superprotonic Conductor $\mathrm{Rb}_{3} \mathrm{H}\left(\mathrm{SO}_{4}\right)_{2}$, Phys. Rev. Lett. 98, 115502 (2007).

[39] K. Hirsch and L. Ojamäe, Quantum-chemical and force-field investigations of ice Ih: Computation of proton-ordered structures and prediction of their lattice energies, J. Phys. Chem. B 108, 15856 (2004).

[40] W. F. Kuhs, C. Sippel, A. Falenty, and T. C. Hansen, Extent and relevance of stacking disorder in ice Ic, Proc. Natl. Acad. Sci. USA 109, 21259 (2012).

[41] T. L. Malkin, B. J. Murray, C. G. Salzmann, V. Molinero, S. J. Pickering, and T. F. Whale, Stacking disorder in ice I, Phys. Chem. Chem. Phys. 17, 60 (2015).

[42] See Supplemental Material at http://link.aps.org/supplemental/ 10.1103/PhysRevB.97.054312 for more information regarding the dependence of the momentum distribution on pressure and temperature, the dependence of the Born effective charges on vibrational displacements, the differences in permittivity between different proton-ordered forms of Ih, and the choice of exchange-correlation functional. The Supplemental Material contains Refs. [31,39,43,44,55-61].

[43] J. Heyd, G. Scuseria, and M. Ernzerhof, Hybrid functionals based on a screened Coulomb potential, J. Chem. Phys. 118, 8207 (2003).

[44] J. Heyd, G. Scuseria, and M. Ernzerhof, Hybrid functionals based on a screened Coulomb potential, J. Chem. Phys. 124, 219906 (2006).

[45] D. L. Wu, W. G. Read, A. E. Dessler, S. C. Sherwood, and J. H. Jiang, UARS/MLS cloud ice measurements: Implications 
for $\mathrm{H}_{2} \mathrm{O}$ transport near the tropopause, J. Atmos. Sci. 62, 518 (2005).

[46] K. Takeya, T. Fukui, R. Takahashi, and K. Kawase, Dielectric constants of $\mathrm{H}_{2} \mathrm{O}$ and $\mathrm{D}_{2} \mathrm{O}$ ice in the terahertz frequency regime over a wide temperature range, J. Opt. 16, 094005 (2014).

[47] S. Fujita, T. Matsuoka, T. Ishida, K. Matsuoka, and S. Mae, A Summary of the Complex Dielectric Permittivity of Ice in the Megahertz Range and its Applications for Radar Sounding of Polar Ice Sheets (Hokkaido University, 2000).

[48] X. Gonze and C. Lee, Dynamical matrices, Born effective charges, dielectric permittivity tensors, and interatomic force constants from density-functional perturbation theory, Phys. Rev. B 55, 10355 (1997).

[49] For instance, the set of 16 eight-molecule proton orderings employed also substantially underestimates the proton-ordering transition temperature of Ih [62].

[50] S. G. Warren and R. E. Brandt, Optical constants of ice from the ultraviolet to the microwave: A revised compilation, J. Geophys. Res. 113, D14220 (2008).

[51] G. Onida, L. Reining, and A. Rubio, Electronic excitations: Density-functional versus many-body Green's-function approaches, Rev. Mod. Phys. 74, 601 (2002).

[52] S. Horiuchi, F. Ishii, R. Kumai, Y. Okimoto, H. Tachibana, N. Nagaosa, and Y. Tokura, Ferroelectricity near room temperature in co-crystals of nonpolar organic molecules, Nat. Mater. 4, 163 (2005).

[53] S. Horiuchi and Y. Tokura, Organic ferroelectrics, Nat. Mater. 7, 357 (2008).
[54] S. Horiuchi, Y. Tokunaga, G. Giovannetti, S. Picozzi, H. Itoh, R. Shimano, R. Kumai, and Y. Tokura, Above-room-temperature ferroelectricity in a single-component molecular crystal, Nature (London) 463, 789 (2010).

[55] J. Klimeš, D. R. Bowler, and A. Michaelides, Chemical accuracy for the van der Waals density functional, J. Phys.: Condens. Matter 22, 022201 (2009).

[56] Z. Wu and R. E. Cohen, More accurate generalized gradient approximation for solids, Phys. Rev. B 73, 235116 (2006).

[57] J. Paier, M. Marsman, K. Hummer, G. Kresse, I. C. Gerber, and J. G. Ángyán, Screened hybrid density functionals applied to solids, J. Chem. Phys. 124, 154709 (2006).

[58] D. M. Ceperley and B. J. Alder, Ground State of the Electron Gas by a Stochastic Method, Phys. Rev. Lett. 45, 566 (1980).

[59] J. P. Perdew and A. Zunger, Self-interaction correction to density-functional approximations for many-electron systems, Phys. Rev. B 23, 5048 (1981).

[60] J. P. Perdew and Y. Wang, Accurate and simple analytic representation of the electron-gas correlation energy, Phys. Rev. B 45, 13244 (1992).

[61] A. Tkatchenko and M. Scheffler, Accurate Molecular Van Der Waals Interactions from Ground-State Electron Density and Free-Atom Reference Data, Phys. Rev. Lett. 102, 073005 (2009).

[62] J.-L. Kuo, The low-temperature proton-ordered phases of ice predicted by $a b$ initio methods, Phys. Chem. Chem. Phys. 7, 3733 (2005). 